\title{
IMPACTO DE NOVAS COMORBIDADES NA QUALIDADE DE VIDA DE OBESOS PÓS-CIRURGIA BARIÁTRICA
}

\author{
IMPACT OF NEW COMORBIDITIES ON THE QUALITY OF LIFE OF OBESE POST- \\ BARIATRIC SURGERY
}

\section{IMPACTO DE NUEVAS COMORBILIDADES EN LA CALIDAD DE VIDA DE OBESOS POST- CIRUGÍA BARIÁTRICA}

Vanessa Souza Lima Verçosa ${ }^{1}$, Maria Alayde Mendonça da Silva², Ivan Romero Rivera $^{3}$, Thays Cristine Ferro Wanderley ${ }^{4}$, Vanessa Reis de Abreu Cavalcante ${ }^{5}$

\section{RESUMO}

Objetivo: analisar a influência das complicações na qualidade de vida de obesos submetidos à cirurgia bariátrica. Método: estudo longitudinal, prospectivo e analítico sobre pacientes obesos, de ambos os gêneros e maiores de 18 anos, em pós-operatório tardio de cirurgia bariátrica. Foram coletados dados demográficos, epidemiológicos e antropométricos. Houve a aplicação de questionário "Impacto do peso sobre a qualidade de vida" para avaliar a qualidade de vida e as complicações observadas no pós-operatório tardio, que foram classificadas em operatórias ou clínicas, de acordo com a classificação listada no protocolo "Bariatric Analysis and Reporting Outcome System- BAROS". Os dados de caracterização da amostra foram analisados por estatística descritiva, obtendose médias e desvio-padrão para cada variável. Os testes Qui-quadrado e Exato de Fisher foram utilizados para a análise das variáveis discretas. Para as variáveis contínuas, que não apresentaram distribuição normal, foi utilizado o teste de Mann-Whitney e teste t de Student, para as amostras pareadas, para a análise das variáveis contínuas com distribuição normal. Resultados: a amostra foi constituída por 50 indivíduos (masculinos,

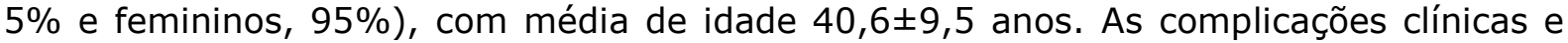
cirúrgicas observadas no pós-operatório atingiram $80 \%$ dos pacientes. Conclusões: a perda de peso por meio da gastroplastia determina a melhora das comorbidades metabólicas, mas as complicações podem influenciar negativamente a qualidade de vida no domínio da "percepção da saúde".

Descritores: Cirurgia Bariátrica; Comorbidade; Obesidade; Qualidade de Vida.

\section{ABSTRACT}

\footnotetext{
1,4 Mestras. Universidade Federal de Alagoas/UFAL. Maceió (AL), Brasil.

${ }^{2,3}$ Doutores. Universidade Federal de Alagoas/UFAL. Maceió (AL), Brasil.

${ }^{5}$ Graduanda de Medicina. Universidade Federal de Alagoas/UFAL. Maceió (AL), Brasil.
}

Rev. Port. Saúde e Sociedade. 2019;4(1): 994 - 1005. 
Objective: to analyze the influence of complications on the quality of life of obese patients submitted to bariatric surgery. Method: longitudinal, prospective and analytical study of obese patients of both genders and over 18 years of age in the late postoperative period of bariatric surgery. Demographic, epidemiological and anthropometric data was collected. The questionnaire "Impact of weight on quality of life" was used to evaluate the quality of life and the complications observed in the late postoperative period that were classified as operative or clinical according to the classification listed in the Bariatric Analysis and Reporting Outcome System- BAROS. The characterization data of the sample was analyzed by descriptive statistics, obtaining means and standard deviation for each variable. Chisquare and Fisher's exact tests were used for the analysis of discrete variables. For the continuous variables that did not present normal distribution, the Mann-Whitney test and the Student $t$ test were used for paired samples for the analysis of continuous variables with normal distribution. Results: the sample consisted of 50 individuals (male, $5 \%$, and female, 95\%), with mean age $40.6 \pm 9.5$ years. The clinical and surgical complications observed in the postoperative period reached $80 \%$ of the patients. Conclusion: weight loss through gastroplasty determines the improvement of metabolic comorbidities, but complications can negatively influence the quality of life in the field of "health perception".

\section{Descriptors: Bariatric Surgery; Comorbidity; Obesity; Quality of life.}

\section{RESUMEN}

Objetivo: analizar la influencia de las complicaciones en la calidad de vida de los obesos sometidos a la cirugía bariátrica. Método: estudio longitudinal, prospectivo y analítico sobre pacientes obesos, de ambos géneros y mayores de 18 años, en postoperatorio tardío de cirugía bariátrica. Se recolectaron datos demográficos, epidemiológicos y antropométricos. Se empleó el cuestionario "Impacto del peso sobre la calidad de vida" para evaluar la calidad de vida y las complicaciones observadas en el postoperatorio tardío que se clasificaron en operatorias o clínicas de acuerdo con la clasificación enumerada en el protocolo "Bariatric Analysis and Reporting Outcome System- BAROS ". Los datos de caracterización de la muestra fueron analizados por estadística descriptiva, obteniéndose medias y desviación estándar para cada variable. Las pruebas Chi-cuadrado y Exacto de Fisher se utilizaron para el análisis de las variables discretas. Para las variables continuadas que no presentaron distribución normal, se utilizaron las pruebas de Mann-Whitney y la prueba t de Student para muestras pareadas para el análisis de las variables continuadas con distribución normal. Resultados: la muestra fue constituida por 50 individuos (masculino, 5\% y femenino, 95\%), con media de edad 40,6 $\pm 9,5$ años. Las complicaciones clínicas y quirúrgicas observadas en el postoperatorio alcanzaron el $80 \%$ de los pacientes. Conclusión: la pérdida de peso a través de la gastroplastia determina la mejora de las comorbilidades metabólicas, pero las complicaciones pueden influenciar negativamente la calidad de vida en el dominio de la "percepción de la salud".

\section{Descriptores: Cirugía Bariátrica; Comorbilidad; Obesidad; Calidad de Vida.}

\section{INTRODUÇÃO}

A obesidade é um problema de saúde pública mundial e pode ser definida como o grau de armazenamento de gordura no organismo associado a riscos para a saúde devido à sua relação com várias complicações metabólicas. ${ }^{1}$ No Brasil, mais de $52,5 \%$ da população (acima de 18 anos) encontra-se acima do peso ideal e, destes, $17,9 \%$ apresentam obesidade. ${ }^{2}$

Rev. Port. Saúde e Sociedade. 2019;4(1): 994 - 1005. 
A cirurgia bariátrica é, na atualidade, a mais efetiva opção para o tratamento da obesidade grave e suas comorbidades. No Brasil, desde 1999, esse procedimento foi incluído na tabela do Sistema de Informações Hospitalares do Sistema Único de Saúde (SIH/SUS). ${ }^{3}$

No estudo realizado no Hospital Universitário da Universidade Federal de Alagoas, utilizando a técnica do bypass gástrico, foi demonstrado o impacto positivo da cirurgia bariátrica na redução de peso, índice de massa corporal (IMC), circunferência abdominal (CA) e frequência de fatores de risco cardiovascular, após seis meses e se mantendo após um ano de realização do procedimento. ${ }^{4}$

Entretanto, apesar dos benefícios oferecidos pela cirurgia bariátrica, há risco de complicações e mortalidade. ${ }^{5} \mathrm{O}$ aparecimento de complicações cirúrgicas e/ou de novas comorbidades pode comprometer a qualidade de vida nesse grupo.

Ao considerar o impacto negativo da obesidade sobre a saúde, o incremento anual no número de gastroplastias realizadas, suas possíveis complicações e a importância de um melhor acompanhamento em longo prazo de obesos submetidos à gastroplastia, este estudo objetiva avaliar a frequência de complicações pós-cirurgia bariátrica e seu impacto na qualidade de vida desses pacientes.

\section{MÉTODO}

Trata-se de um estudo longitudinal, prospectivo e analítico. A amostragem foi realizada por conveniência com pacientes em pós-operatório tardio entre dois a quatro anos de cirurgia bariátrica.

Os critérios de inclusão foram: pacientes submetidos à gastroplastia, de ambos os sexos, maiores de 18 anos, com acompanhamento no ambulatório de Cardiologia de um hospital universitário. Quem não apresentou os critérios de inclusão foi excluído da amostra.

Todos os pacientes foram submetidos ao mesmo tipo de cirurgia, por meio da técnica de bypass gástrico, por via convencional, realizada pela equipe de cirurgia bariátrica do hospital, submetendo-os ao mesmo protocolo de pesquisa, que foi dividido em quatro etapas.

Na primeira etapa, foi realizada a análise do banco de dados geral da linha de pesquisa de "Avaliação cardiológica em obesos com indicação de gastroplastia", do Serviço de Cardiologia do Hospital Universitário, para a identificação dos

Rev. Port. Saúde e Sociedade. 2019;4(1): 994 - 1005. 
pacientes que contemplavam os critérios de inclusão. Durante o atendimento ambulatorial, os pacientes foram convidados a participar da pesquisa e assinaram o Termo de Consentimento Livre e Esclarecido (TCLE).

$\mathrm{Na}$ segunda etapa, foi consultado o banco de dados para a coleta de informações do período de pré-operatório de cada paciente para a identificação dos dados demográficos (gênero, idade, escolaridade e estado civil), epidemiológicos (comorbidades e qualidade de vida) e antropométricos (Índice de Massa Corporal - IMC, peso, altura e circunferência abdominal - CA).

Na terceira etapa, foi realizada a análise de prontuários para a estruturação do novo banco de dados, com informações do intraoperatório e do pós-operatório do grupo estudado. Foram coletados dados epidemiológicos e antropométricos.

$\mathrm{Na}$ quarta etapa, houve a aplicação do questionário de qualidade de vida e a coleta de dados antropométricos atuais, dosagens bioquímicas, utilização atual de medicamentos (para caracterizar a presença de comorbidades) e submissão a novos procedimentos cirúrgicos.

Na aferição do peso e altura, foi utilizada a balança mecânica de fabricação da empresa WELMY e procedência nacional. O paciente era orientado a retirar os calçados e roupas antes de realizar a medida do peso. Para a medida da circunferência abdominal (CA), foi verificado o maior perímetro abdominal entre a última costela e a crista ilíaca, segundo recomendação da Organização Mundial de Saúde (OMS). A partir do peso e altura, foi calculado o valor do IMC (divisão do peso corporal em quilogramas pelo quadrado da altura em metros) para cada paciente e utilizada sua classificação para obesidade grau I (IMC $=30,0$ a $\left.34,9 \mathrm{~kg} / \mathrm{m}^{2}\right)$, II $\left(\right.$ IMC $=35,0$ a $\left.39,9 \mathrm{~kg} / \mathrm{m}^{2}\right)$ e III $\left(\right.$ IMC $\left.>40 \mathrm{~kg} / \mathrm{m}^{2}\right){ }^{6}$

Para o diagnóstico de comorbidades, foram utilizados os parâmetros constantes na I Diretriz Brasileira de Diagnóstico e Tratamento da Síndrome Metabólica. $^{7}$

As complicações observadas no pós-operatório tardio da cirurgia bariátrica foram classificadas em operatórias ou clínicas de acordo com a classificação listada no protocolo "Bariatric Analysis and Reporting Outcome System-BAROS". 8

O instrumento utilizado para avaliar a qualidade de vida no pré e no pósoperatório foi o questionário "Impact on Weight on Quality of life-Lite (IWQOLLite)", que é um questionário com 31 itens sobre a qualidade de vida relacionada com o peso, agrupados em cinco domínios (função física, autoestima, vida sexual, constrangimento público e trabalho), havendo pontuações para todos os itens e

Rev. Port. Saúde e Sociedade. 2019;4(1): 994 - 1005. 
escore total de zero a 100. O escore mais baixo indica maior comprometimento da qualidade de vida. ${ }^{9}$

Os dados de caracterização da amostra foram analisados por estatística descritiva, obtendo-se média e desvios-padrão. Foi utilizado teste t de Student para amostras pareadas de variáveis contínuas com distribuição normal e o teste Qui-quadrado ou Exato de Fisher para a análise das variáveis discretas. Para as variáveis contínuas que não apresentaram distribuição normal, foi utilizado o teste de Mann-Whitney. O nível de significância adotado foi de $5 \%$ e todas as análises foram realizadas utilizando o software BioEstat, versão 5.3.

Todos os indivíduos assinaram o Termo de Consentimento Livre e Esclarecido (TCLE). A pesquisa foi aprovada pelo Comitê de Ética em Pesquisa da UFAL sob o número do CAAE: 42 778914.3.0000.5013.

\section{RESULTADOS}

No período de 2004 a 2015, foi encaminhado, para o ambulatório de Cardiologia, um total de 268 obesos, com indicação de gastroplastia, para a avaliação cardiológica pré-operatória. Destes, 50 indivíduos estavam entre dois e quatro anos de pós-operatório tardio (2011-2013). A amostra foi constituída de dois pacientes do gênero masculino (5\%) e 48 do gênero feminino (95\%), com idades entre 23 e 61 anos, média de 40,66 ( $\pm 9,47)$, com grau de escolaridade de mais de oito anos de estudo (64\%).

Ao utilizar a classificação da obesidade por meio do IMC, observou-se que, no período pré-operatório, $98 \%$ dos pacientes apresentaram obesidade III e, no pós-operatório tardio, apenas $8 \%$ dos pacientes continuaram nesse grupo, havendo significância estatística nessa mudança de perfil antropométrico (Tabela 1).

Tabela 1. Grau de obesidade determinado pelo Índice de Massa Corporal (IMC) no período pré-operatório e pós-operatório de cirurgia bariátrica.

\begin{tabular}{|c|c|c|c|c|}
\hline IMC $\left(\mathrm{kg} / \mathrm{m}^{2}\right)$ & Classificação & $\begin{array}{c}\text { Pré- } \\
\text { operatório } \\
\text { N }(\%)\end{array}$ & $\begin{array}{c}\text { Pós- } \\
\text { operatório } \\
\text { N (\%) }\end{array}$ & $\mathbf{P}$ \\
\hline $\begin{array}{l}\text { Abaixo de } 25 \\
25 \mid-29.9 \\
30 \mid-34.9 \\
35 \mid-39.9 \\
\text { Acima de } 40\end{array}$ & $\begin{array}{c}\text { Normal } \\
\text { Sobrepeso } \\
\text { Obesidade I } \\
\text { Obesidade II } \\
\text { Obesidade III } \\
\end{array}$ & $\begin{array}{c}0(0,0) \\
0(0,0) \\
0(0,0) \\
1(2,0) \\
49(98,0)\end{array}$ & $\begin{array}{c}2(4,0) \\
8(16,0) \\
29(58,0) \\
7(14,0) \\
4(8,0)\end{array}$ & $\begin{array}{c}0,2057 * * \\
0,0023 * i \\
<0,0001^{*} i \\
0,0103^{*} \\
<0,0001^{*} \\
\end{array}$ \\
\hline
\end{tabular}

Rev. Port. Saúde e Sociedade. 2019;4(1): 994 - 1005. 
As principais comorbidades presentes no pré-operatório mantiveram-se após a cirurgia bariátrica, mas, em frequência menor, com diminuição estatisticamente significante dessas frequências, conforme demonstrado na tabela 2.

Foi utilizado teste Qui-quadrado*; Exato de Fisher**; ${ }^{i}$ refere-se à diferença estatisticamente significante $(p<0,05)$

\section{Tabela 2. Frequência das comorbidades no período pré-operatório e no pós-operatório tardio da cirurgia bariátrica.}

\begin{tabular}{lccc}
\hline \multicolumn{1}{c}{ Variáveis } & $\begin{array}{c}\text { Pré-operatório } \\
\text { N }(\%)\end{array}$ & $\begin{array}{c}\text { Pós-operatório } \\
\text { N }(\%)\end{array}$ & P \\
\hline DM & $11(22,0)$ & $5(10,0)$ & $0,1714^{* *}$ \\
Dislipidemias & $19(38,0)$ & $3(6,0)$ & $0,0003^{* i}$ \\
HAS & $35(70,0)$ & $18(36,0)$ & $0,0028^{* i}$ \\
SM & $37(74,0)$ & $13(26,0)$ & $<0,0001^{* i}$ \\
Sem comorbidades & $6(12,0)$ & $21(42,0)$ & $0,0016^{*} ;$ \\
\hline
\end{tabular}

HAS: Hipertensão Arterial Sistêmica; DM: Diabetes Mellitus; SM: Síndrome Metabólica. Foi utilizado teste Qui-quadrado* ;Exato de Fisher**; ${ }^{\dagger}$ refere-se à diferença estatisticamente significativa $(p<0,05)$

As complicações e novas comorbidades observadas no pós-operatório tardio da cirurgia bariátrica ocorreram em 40 pacientes (80\%) e apenas dez pacientes (20\%) não as apresentaram. As novas comorbidades foram classificadas em complicações operatórias e complicações clínicas. As principais complicações operatórias foram a hérnia incisional (36\%) e a colelitíase (28\%); as complicações clínicas foram vômitos (42\%) e perda de cabelo (20\%). A distribuição dessas complicações é apresentada na tabela 3.

\section{Tabela 3. Frequência absoluta e relativa das complicações e novas comorbidades no pós-operatório tardio de cirurgia bariátrica.}

\begin{tabular}{lrc}
\hline \multicolumn{1}{c}{ Variável } & N & $(\mathbf{\%})$ \\
\hline Complicações Operatórias & & 36 \\
Hérnia incisional & 18 & 28 \\
Colelitíase & 14 & 2 \\
Deiscência de sutura & 1 & 4 \\
Obstrução intestinal & 2 & 2 \\
Fístula gastrogástrica & 1 & 2 \\
Estenose de Anastomose & 1 & 2 \\
Esofagite de refluxo & 1 & 2 \\
Complicações Clínicas & & 4 \\
Pneumonia & 1 & 42 \\
Depressão severa & 2 & 21 \\
Vômitos & &
\end{tabular}

Rev. Port. Saúde e Sociedade. 2019;4(1): 994 - 1005. 
A tabela 4 demonstra as médias e o desvio-padrão do escore para cada domínio do questionário do grupo de pacientes avaliados, comparando-se os períodos pré e pós-operatório tardio.

Tabela 4. Valores das médias e desvio padrão de cada domínio, referentes à qualidade de vida (IWQOL-Life) dos pacientes no pré e no pós-operatório tardio da cirurgia bariátrica.

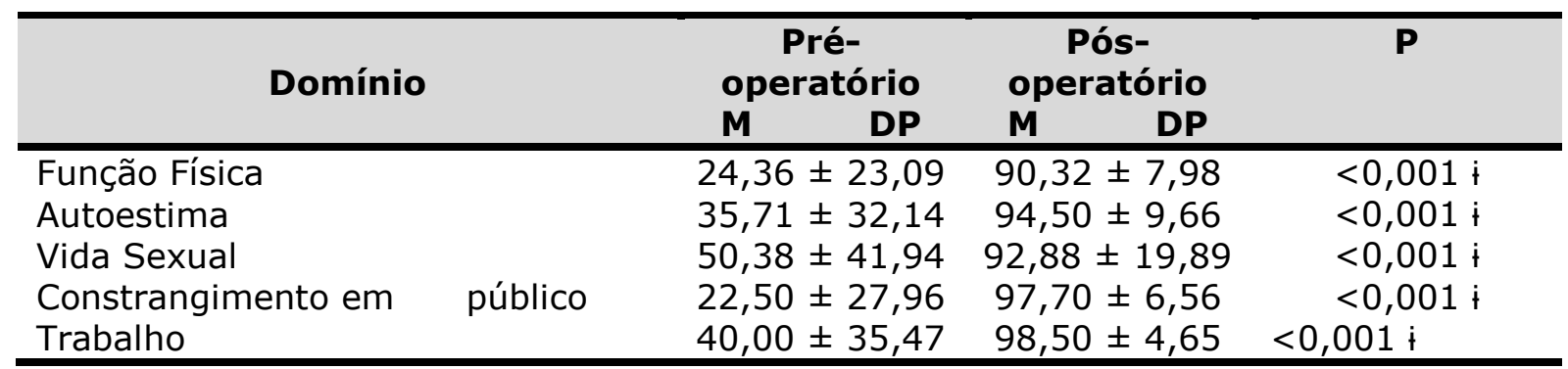

Foi utilizado teste Mann-Whitney; ${ }^{i}$ refere-se à diferença estatisticamente significativa $(p<0,05)$

$\mathrm{Na}$ tabela 5, podem ser observadas médias e desvio-padrão do escore, comparando os pacientes que apresentaram complicações (operatórias e clínicas) e os que não apresentaram, havendo diferença estatisticamente significante no domínio função física $(p<0,003)$.

Tabela 5. Valores das médias e desvio padrão de cada domínio, referentes à qualidade de vida (IWQOL-Life) dos pacientes com e sem complicações no pós-operatório tardio da cirurgia bariátrica.

\begin{tabular}{|c|c|c|c|}
\hline Domínio & $\begin{array}{c}\text { Com } \\
\text { complicações } \\
M\end{array}$ & $\begin{array}{c}\text { Sem } \\
\text { complicações } \\
\text { M DP }\end{array}$ & $\mathbf{P}$ \\
\hline Função Física & $88,40 \pm 8,39$ & $94,88 \pm 4,65$ & $0,003 * * i$ \\
\hline Autoestima & $93,65 \pm 10,39$ & $96,42 \pm 7,61$ & $0,199 *$ \\
\hline Vida Sexual & $92,28 \pm 21,76$ & $94,27 \pm 12,90$ & $0,383 *$ \\
\hline Constrangimento em público & $97,37 \pm 7,41$ & $98,75 \pm 2,26$ & $0,472 * *$ \\
\hline Trabalho & $98,10 \pm 5,29$ & $99,47 \pm 1,80$ & $0,324 * *$ \\
\hline
\end{tabular}

Foi utilizado teste $\mathrm{t}$ de Student * para variáveis que apresentaram distribuição normal e Mann-Whitney** para as que não apresentaram; ${ }^{i}$ refere-se à diferença estatisticamente significativa $(p<0,05)$

\section{DISCUSSÃO}

Rev. Port. Saúde e Sociedade. 2019;4(1): 994 - 1005. 
Neste estudo, a maioria dos pacientes submetidos à cirurgia bariátrica foi do gênero feminino, uma característica também evidenciada em outros estudos, por haver maior motivação das mulheres obesas do que dos homens obesos em perder peso, em consequência das pressões sociais a respeito da estética. ${ }^{10-1}$

O nível de escolaridade elevado também é um fator determinante para a busca do tratamento, pois contribui na persistência e escolha por estratégias para conseguir alternativas terapêuticas para manter o peso, como também proporciona melhor compreensão das informações fornecidas durante as consultas e intervenções educativas. ${ }^{12}$

A cirurgia bariátrica é considerada bem-sucedida se houver perda de, no mínimo, 50\% do peso excedente no momento da operação e se o indivíduo apresentar IMC fora da classificação de obesidade III. ${ }^{13}$ Foi observada uma redução importante na média do peso corporal, que passou de $121 \mathrm{Kg}$ para $83 \mathrm{Kg}$, enquanto a média do IMC passou de $48 \mathrm{~kg} / \mathrm{m}^{2}$ para $33 \mathrm{~kg} / \mathrm{m}^{2}$, o que representou uma transição estatisticamente significante na classificação da obesidade III de $98 \%$ dos pacientes no período pré-operatório para apenas $4 \%$ no pós-operatório.

Em relação às comorbidades (hipertensão arterial sistêmica, Diabetes Mellitus, dislipidemia e síndrome metabólica), ficou evidenciada a redução importante em sua frequência, refletindo uma melhora significante na saúde clínica dos indivíduos operados. No estudo Swedish Obese Subjects (SOS), coorte de 14 anos de seguimento, evidenciou-se que a cirurgia bariátrica foi associada à redução do número de mortes por doenças cardiovasculares e menor incidência de eventos cardiovasculares em adultos obesos. ${ }^{14}$

Apesar da incontestável melhoria das comorbidades metabólicas no pósoperatório da cirurgia bariátrica, há uma preocupação com a possibilidade de ocorrência de complicações precoces e tardias, havendo uma variação nas taxas de incidência das mesmas, nos diferentes estudos. As complicações imediatas ocorreram em 9,6\% dos pacientes no pós-operatório, e relataram uma taxa de complicação de $17 \%$ e taxa de reoperação de $7 \%$, sendo o bypass gástrico o mais eficaz na perda de peso, apesar de mais associado às complicações. ${ }^{10,15}$

A maioria dos pacientes evoluiu com complicações (operatórias e/ou clínicas), representando $80 \%$ da amostra. Isso pode estar relacionado a alguns fatores como o tipo de via utilizada (laparotomia) ou a dificuldade em fazer um diagnóstico precoce das complicações, pelo excesso de peso ainda presente ou

Rev. Port. Saúde e Sociedade. 2019;4(1): 994 - 1005. 
pela dificuldade da realização de exames de imagem no cenário do Sistema Único de Saúde.

A principal complicação operatória encontrada foi hérnia incisional (36\%), que é comum em cirurgias abdominais e principalmente após a cirurgia bariátrica aberta. Outros estudos apresentaram menores taxas desta complicação, o que pode ser explicado pela crescente utilização de técnicas minimamente invasivas que diminuam a incidência desta complicação. ${ }^{16-8}$

A colelitíase ocorreu em $28 \%$ dos pacientes do estudo, uma taxa maior quando comparada a de outros estudos realizados no país, com taxas de $12,1 \%$ e $15,3 \%$, mas que também demonstram a colelitíase como uma complicação prevalente nos pacientes no pós-operatório de cirurgia bariátrica e que também necessita, como a hérnia incisional, de um novo manejo hospitalar. ${ }^{19-20}$

As complicações clínicas mais frequentes foram vômitos (42\%) e perda de cabelo (20\%), que avaliaram qualitativamente as complicações cirúrgicas e clínicas dos pacientes após a cirurgia, usando, como referência, o sistema formal de classificação do protocolo BAROS e detectando, entre as manifestações clínicas pós-operatórias, mudanças relacionadas à ingestão de alimentos, tais como náuseas, vômitos e perda de cabelo, no grupo que utilizou a técnica de bypass gástrico. ${ }^{21}$ Estes dados demonstram que as complicações pós-operatórias são frequentes, exigindo que os profissionais estejam preparados para fazer 0 diagnóstico e o tratamento precoces.

Antes da cirurgia e em função do excesso de peso, os indivíduos demonstraram ter uma qualidade de vida gravemente prejudicada em todos os domínios avaliados (função física, autoestima, relações sociais, relações profissionais e vida sexual). Após a cirurgia bariátrica, observou-se a modificação significante no escore de avaliação da qualidade de vida em todos os domínios, com percepção dos avaliados de uma melhor qualidade de vida. Isso ratifica a importância da cirurgia bariátrica como procedimento eficaz para o tratamento da obesidade, por determinar importante redução do peso e melhora das comorbidades, gerando, como consequência, uma percepção de melhoria na qualidade de vida, em todos os domínios avaliados.

Este estudo corroborou com o que compararam o impacto da perda de peso e da recuperação do peso perdido na qualidade de vida do obeso, utilizando o IWQOL-Lite em intervalos de três meses. Esses autores observaram que a

Rev. Port. Saúde e Sociedade. 2019;4(1): 994 - 1005. 
qualidade de vida melhorou, na mesma proporção, para cada unidade de peso perdido e deteriorou para cada unidade de peso recuperada. ${ }^{12}$

A cirurgia bariátrica demonstra ser um procedimento eficaz no tratamento da obesidade mórbida e no controle das comorbidades e que a qualidade de vida foi avaliada de forma positiva em $93,2 \%$ dos pacientes por meio do protocolo BAROS. ${ }^{18}$

Utilizando o protocolo BAROS para a sua avaliação, constataram que $79 \%$ dos pacientes no pós-operatório tardio da cirurgia bariátrica afirmaram melhora significativa da qualidade de vida após a cirurgia. Apesar da melhoria identificada na qualidade de vida, quando são comparados os indivíduos no pré-operatório e no pós-operatório, quando se analisa, no pós-operatório, o grupo de pacientes que evoluiu com alguma complicação (clínica e/ou cirúrgica) em relação aos que não a apresentaram, observa-se que a percepção de melhora na qualidade se mantém em todos os domínios, exceto naquele da função física. Esse domínio permite, ao paciente, avaliar sua qualidade de vida quanto à percepção da sua própria saúde que, nesse caso, se apresenta comprometida pela ocorrência de novas situações que exigem tratamento clínico e/ou cirúrgico. ${ }^{12}$

Quando a perda de peso estabiliza, mas os sintomas digestivos persistem e novas operações pelas complicações são necessárias, essas situações são encaradas como novas comorbidades, determinando, assim, um impacto negativo na qualidade de vida que havia melhorado substancialmente com a perda de peso.

\section{CONCLUSÃO}

Os resultados demonstram que o tratamento da obesidade não é concluído quando a perda máxima de peso é obtida, considerando que a ocorrência de novas condições que exigem intervenções clínicas e cirúrgicas comprometem a saúde e a percepção de saúde do paciente. Assim, o planejamento pré-operatório, com a escolha de técnicas que diminuam a ocorrência de complicações precoces e/ou tardias, é mandatório nos programas que atendem obesos graves e os preparam para a gastroplastia. É necessário incorporar, no manuseio desses pacientes, a compreensão da importância da sua avaliação multidimensional, muito evidente na percepção dos mesmos sobre a sua qualidade de vida que, com a perda de peso, melhora consideravelmente em todos os domínios, mas fica comprometida na percepção de "sentir-se com saúde", quando a perda de peso é acompanhada de novas doenças.

Rev. Port. Saúde e Sociedade. 2019;4(1): 994 - 1005. 


\section{REFERÊNCIAS}

1. World Health Organization. Obesity: Preventing and Managing the Global Epidemic [Internet]. Geneva: WHO; 2000 [cited 2018 Aug 10]. Available from: https://www.who.int/nutrition/publications/obesity/WHO_TRS_894/en/

2. Ministério da Saúde (BR), Gabinete do Ministro. Portaria n. 196, de 29 de fevereiro de 2000. Aprova os critérios clínicos para a indicação de realização de gastroplastia como tratamento cirúrgico da obesidade mórbida, no âmbito do Sistema Único de Saúde [Internet]. Brasília: Ministério da Saúde; 2000 [cited 2018 Aug 08]. Available from: http://www.jusbrasil.com.br/diarios/1061523/pg-32-secao-1-diario-oficialda-uniao-dou-de-01-03-2000.

3. Ministério da Saúde (BR), Secretaria De Vigilância em Saúde, Departamento de Vigilância de Doenças e Agravos Não Transmissíveis e Promoção da Saúde. Vigitel - Vigilância de Fatores de Risco e Proteção para Doenças Crônicas por Inquérito Telefônico [Internet]. Brasília: Ministério da Saúde; 2014 [cited 2018 Aug 01]. Available http://bvsms.saude.gov.br/bvs/publicacoes/vigitel_brasil_2014.pdf

from:

4. Silva MAM, Rivera IR, Barbosa EMW, Crispim MAC, Farias GC, Fontan AJA, et al. Frequency of cardiovascular risk factors before and 6 and 12 months after bariatric surgery. Rev Assoc Med Bras. 2013 July/Aug; 59(4):381-6. Doi: http://dx.doi.org/10.1016/j.ramb.2013.02.009

5. Smith MD, Patterson E, Wahed AS, Belle SH, Berk PD, Courcoulas AP, et al. Thirtyday Mortality after Bariatric Surgery: Independently Adjudicated Causes of Death in the Longitudinal Assessment of Bariatric Surgery. Obes Surg. 2011 Nov; 21(11):1687-92. Doi: 10.1007/s11695-011-0497-8.

6. World Health Organization. Physical status: the use and interpretation of anthropometry [Internet]. Geneva:WHO;1995. Available From: https://www.who.int/childgrowth/publications/physical_status/en/

7. Brandão $A P$, Brandão $A A$, Nogueira AR, Suplicy $H$, Guimarães JI, Oliveira JEP. I Diretriz Brasileira de Diagnóstico e Tratamento da Síndrome Metabólica. Arq Bras Cardiol [Internet]. $2005 \mathrm{Apr}$ [cited 2018 Aug 01]; 84(Suppl 1):1-28. Available from: http://publicacoes.cardiol.br/consenso/2005/dir_resumida.pdf

8. Oria $\mathrm{H}$, Moorehead MK. Bariatric analysis and reporting outcome system (BAROS). Obes Surg. 1998 Oct; 8(5): 487-99. Doi: 10.1381/096089298765554043.

9. Engel SG, Kolotkin RL, Teixeira PJ, Sardinha LB, Vieira PN, Palmeira AL, et al. Psychometric and cross-national evaluation of a Portuguese version of the Impact of Weight on Quality of Life-Lite (IWQOL-Lite) questionnaire. Eur Eat Disorders Rev. 2005 Mar; 13(2):133-43. Doi: https://doi.org/10.1002/erv.614

10. Santo MA, Pajecki D, Riccioppo D, Cleva R, Kawamoto F, Cecconello I. Early complications in bariatric Surgery: incidence, diagnosis and treatment. Arq Gastroenterol. [Internet] 2013 Jan/Mar [cited 2018 Sept 06]; 50(1):50-5. Available from: https://www.ncbi.nlm.nih.gov/pubmed/23657307

11. Pona AA, Heinberg LJ, Lavery M, Ben-Porath YS, Rish J M. Psychological predictors of body image concerns 3 months after bariatric surgery. Surg obes relat dis. 2016 Jan; 12(1):188-93. Doi: 10.1016/j.soard.2015.05.008

Rev. Port. Saúde e Sociedade. 2019;4(1): 994 - 1005. 
12. Barros LM, Moreira RAN, Frota NM, Caetano JA. Changes in Quality of Life After Bariatric Surgery. J Nurs UFPE online. 2013 May; 7(5):1365-75. Doi: 10.5205/reuol.3960-31424-1-SM.0705201315

13. Associação Brasileira para o Estudo da Obesidade e da Síndrome Metabólica. Diretrizes brasileiras de obesidade 2009/2010/ABESO Associação Brasileira para o Estudo da Obesidade e da Síndrome Metabólica [Internet]. Itapevi: ABESO; 2009 [cited 2018 Mar 26]. Available From: http://www.abeso.org.br/pdf/diretrizes_brasileiras_obesidade_2009_2010_1.pdf

14. Sjöström L, Peltonen $M$, Jacobson $P$, Sjöström CD, Karason $K$, Wedel $H$, et al . Bariatric Surgery and Long-term Cardiovascular. JAMA. 2012 Jan; 307(1):5665. Doi: 10.1001/jama.2011.1914.

15. Chang SH, Stoll CRS, Song J, Varela JE, Eagon CJ, Colditz GA. Bariatric surgery: an updated systematic review and meta-analysis, 2003-2012. JAMA Surg. 2014 Mar; 149(3) :275-87. Doi: 10.1001/jamasurg.2013.3654

16. Podnos YD, Jimenez JC, Wilson SE, Stevens CM, Nguyen NT. Complications after laparoscopic gastric bypass: a 3464 case review. Arch Surg. 2003 Sept; 138:95761. Doi: 10.1001/archsurg.138.9.957

17. Healy P, Clarke C, Reynolds I, Arumugasamy M, McNamara D. Complications of bariatric surgery-What the general surgeon needs to know. Surgeon. 2016 Apr;14(2):91-8.http: Doi: 10.1016 / j.surge.2015.08.003

18. Castanha CR, Ferraz AAB, Castanha AR, Belo GQMB, Lacerda RMR, Vilar L. Evaluation of quality of life, weight loss and comorbidities of patients undergoing bariatric surgery. Rev Col Bras Cir. 2018 July; 45(3):e1864. Doi: http://dx.doi.org/10.1590/0100-6991e-20181864

19. Vieira E, Iser BPM. Complications due to bariatric surgery in patients attended in a hospital in southern santa catarina. ACM arq catarin med [Internet]. 2018 July/Sept $\begin{array}{lllll}\text { [cited } 2018 & \text { Dec 27]; 47(3):74-84. }\end{array}$ from: http://www.acm.org.br/acm/seer/index.php/arquivos/article/view/373/275

20. Wrzesinski A, Corrêa JM, Fernandes TMB, Monteiro LF, Trevisón FS, Complications requiring hospital management after bariatric surgery. $A B C D$, Arq bras cir escavação. 2015; 28(Suppl 1):03-06. Doi: /10.1590/S0102$6720201500 S 100003$

21. Duarte MI, Bassitt DP, Azevedo OC, Waisberg J, Yamaguchi N, Pinto Junior PE. Impact on quality of life, weight loss and comorbidities: a study comparing the biliopancreatic diversion with duodenal switch and the banded Roux-Y gastric bypass. Arq Gastroenterol. 2014 Oct; 51(4):320-27. Doi: 10.1590/S000428032014000400010

Rev. Port. Saúde e Sociedade. 2019;4(1): 994 - 1005. 